\title{
An interactive tool for teaching map projections
}

\section{Other Conference Item}

\section{Author(s):}

Heitzler, Magnus; Bär, Hans Rudolf; Schenkel, Roland; Hurni, Lorenz

Publication date:

2018

Permanent link:

https://doi.org/10.3929/ethz-b-000317855

\section{Rights / license:}

Creative Commons Attribution 4.0 International

\section{Originally published in:}

https://doi.org/10.7287/peerj.preprints.27218v1 


\section{An interactive tool for teaching map projections}

Map projections are one of the fundamental concepts of geographic information science and cartography. An understanding of the different variants and properties is critical when creating maps or carrying out geospatial analyses. To support learning about map projections, we present an online tool that allows to interactively explore the construction process of map projections. A central 3D view shows the three main building blocks for perspective map projections: the globe, the projection surface (cone, cylinder, plane) and the projection center. Interactively adjusting these objects allows to create a multitude of arrangements forming the basis for common map projections. Further insights can be gained by adding supplementary information, such as projection lines and Tissot's indicatrices. Once all objects have been arranged in a desired way, the projection surface can be unrolled to form the final flat map. Currently, the tool is limited to visualize the construction of true perspective map projections. In the future, prime concerns are to increase the genericity of the application to support more map projections and to integrate it into the GITTA (Geographic Information Technology Training Alliance) platform. 


\title{
An Interactive Tool for Teaching Map Projections
}

\author{
Magnus Heitzler ${ }^{1}$, Hans-Rudolf Bär ${ }^{1}$, Roland Schenkel ${ }^{2}$, and Lorenz \\ Hurni $^{1}$
}
${ }^{1}$ Institute of Cartography and Geoinformation, ETH Zurich, Zurich, Switzerland ${ }^{2}$ ESRI Schweiz AG, Zurich, Switzerland

\author{
Corresponding author: \\ Magnus Heitzler ${ }^{1}$ \\ Email address: hmagnus@ethz.ch
}

\begin{abstract}
Map projections are one of the fundamental concepts of geographic information science and cartography. An understanding of the different variants and properties is critical when creating maps or carrying out geospatial analyses. To support learning about map projections, we present an online tool that allows to interactively explore the construction process of map projections. A central 3D view shows the three main building blocks for perspective map projections: the globe, the projection surface (cone, cylinder, plane) and the projection center. Interactively adjusting these objects allows to create a multitude of arrangements forming the basis for common map projections. Further insights can be gained by adding supplementary information, such as projection lines and Tissot's indicatrices. Once all objects have been arranged in a desired way, the projection surface can be unrolled to form the final flat map. Currently, the tool is limited to visualize the construction of true perspective map projections. In the future, prime concerns are to increase the genericity of the application to support more map projections and to integrate it into the GITTA (Geographic Information Technology Training Alliance) platform.
\end{abstract}

\section{INTRODUCTION}

Map projections are a key concept in cartography and geographic information science. Choosing an inappropriate map projection may cause severely flawed results when carrying out geospatial analyses or may distort a map reader's view on the world when exploring a thematic or topographic map. Hence, a thorough understanding of the construction process and map properties is important. While mapping software typically implement map projections as a set of equations (e.g., kartograph.js (Aisch, 2014) or d3.js (Bostock et al., 2011)), educational resources follow a more graphical approach to explain the underlying geometrical meaning of the construction process. For example, the construction process for the Lambert conformal conic projection is explained in the GITTA (Geographic Information Technology Training Alliance) learning platform using the two illustrations given in Figure 1 (GITTA, 2018). On the left, a cone surrounding the globe is sketched, touching it at the standard parallel. On the right, it is indicated how the final 2D map looks like, which is obtained by flattening the cone. Similar depictions can be found in other sources such as textbooks and manuals (e.g., those by Iliffe and Lott (2008) and Snyder (1987)). Such depictions give a better impression of the construction process than mathematical formulas. However, they only show snapshots of an actually continuous procedure and thus leave considerable mental workload to the reader. In this paper, we present a tool, which allows to explore this process in a more flexible way.

\section{PROJECTION TOOL}

The educational tool for the construction of map projections is an interactive 3D web application that enables the user to explore the map construction process in a comprehensive and interactive manner It consists of a central 3D view in which the different arrangements of the required objects are shown:

\footnotetext{
${ }^{1}$ The prototype can be tested at https://gevian.github.io/GITTA-MP/
} 

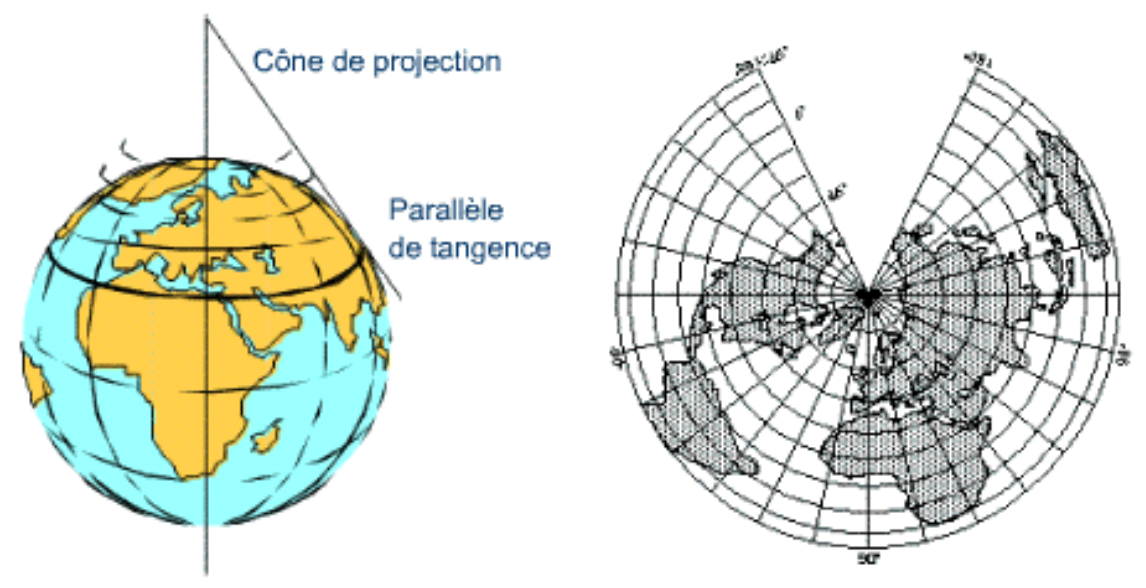

Figure 1. Typical illustrations on the map construction process exemplified for Lambert's conformal conic projection. Left: 2D illustration of the positioning of the cone and the globe to carry out the projection process. Right: Illustration of the final 2D map obtained by flattening the cone. Source: GITTA (2018).

\begin{abstract}
The globe (simplified as a unit sphere), the projection surface and the projection center. Two examples on possible arrangements in the 3D scene are given in Figure 2 The projection center is represented as an orange sphere to follow the light bulb metaphor when explaining map projections: Rays are being sent out from this point light source, intersecting the globe and hitting the projection surface. Such a ray is displayed in red color on the left side of Figure 2. The color on the projection surface is determined by the color where the ray intersects the globe, thus giving a direct impression of how features on the surface of the globe are represented on the projection surface. The shape of the projection surface and the positioning of the projection center can be interactively manipulated.
\end{abstract}
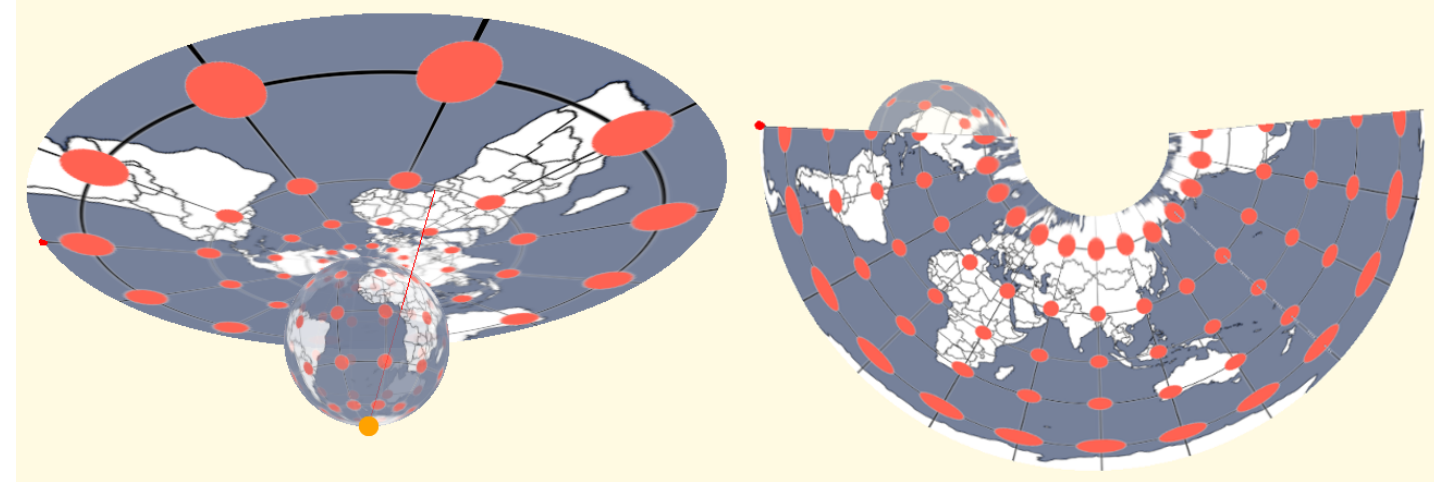

Figure 2. Different configurations of map projections as visualized in the 3D view. Left: the stereographic azimuthal projection constructed by placing the plane on the North pole and setting the projection center to the South pole. Right: a conical projection has been constructed and subsequently unrolled to obtain the flat map.

Traditionally, three main projection surfaces are described in the literature: the plane, the cylinder and the cone. Each of these surfaces has distinct properties, such as its standard parallels, its orientation around the globe, etc. Rather than providing different interfaces for each of these surfaces, we exploit 
the fact that these surfaces are special cases of the conical frustum. Each of them can be constructed by modifying the frustum's upper and lower radii, height and position in respect to the globe. This simplifies the user interface and at the same time makes this relationship obvious. The corresponding parameters are shown on the left side of Figure 3.

The position of the projection center is critical for map projections. It typically resides anywhere along the local y-axis of the projection surface. Hence, the position of the projection center is directly coupled to the orientation of the projection surface. For rare cases, such as the oblique perspective projection, it is possible to move the projection center away from the y-axis, using the respective sliders for relative latitude and longitude. To create orthographic projections, the projection center needs to be positioned at positive or negative infinity, which can be approximated with high or low numerical values (e.g., \pm 100$)$.

Finally, the projection surface can be unrolled, which is visualized using a smooth animation, resulting in the flat map. One result of this process is depicted in Figure 2 on the right. This final representation typically completes the map construction process. If other configurations are to be investigated, it is possible to roll the map again. Apart from the standard texture depicting the world's borders and land areas, it is possible to include a graticule and Tissot's indicatrices to investigate distortions.
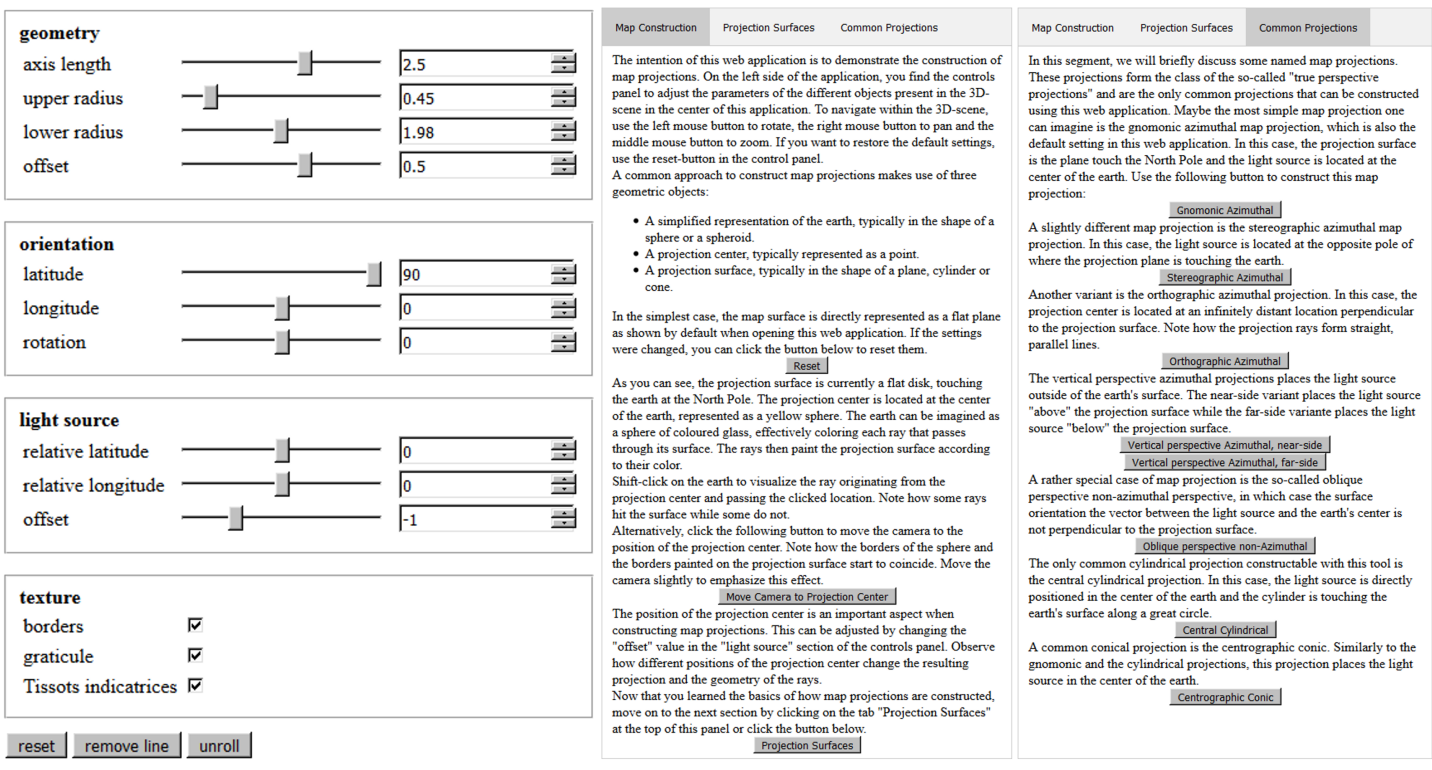

Figure 3. Controls and tutorial of the projection tool. Left: User interface elements to explore different configurations of map projections. Center: The first page of the accompanying tutorial. Right: Brief explanations on common map projections along with buttons to construct them automatically.

The general controls allow for an unguided exploration of the map construction process. A more focused approach is included in the form of a tutorial, which explains general concepts and guides the user in adjusting certain parameters or showcasing relationships using pre-defined buttons. The tutorial is accompanied with a list of common projections that can be constructed with the tool. Shortcuts are provided that automatically adjust the parameters to obtain the respective map projection.

\section{OUTLOOK}

The current version of the projection tool is limited in several ways. First, only true perspective map projections can be directly generated by constructing straight lines originating from a single projection center. Examples for currently unsupported map projection are those requiring the construction of circular arcs instead of straight lines, such as the azimuthal equidistant projection, and those requiring to scale the resulting flat map, such as the Mercator projection. Second, the graphical quality of the constructed map projections does not suffice cartographic standards. Blurriness and other artifacts are common. Third, the user interface and the tutorial need to be improved to provide a better learning experience. These limitations will be addressed in next versions of the projection tool. In the near future, the focus will be 
to improve the tutorial, clean up the user interface and improve the robustness of the tool to ensure its smooth integration into the GITTA platform.

\section{CONCLUSION}

Allowing to interactively construct map projections in a 3D environment as provided by the described projection tool goes beyond the common static approaches provided by conventional educational resources. Hence, it is expected that the projection tool will be a valuable add-on to the GITTA learning platform. However, remaining shortcomings, such as its restriction to true perspective map projections limit its use. To overcome these, it is aimed to continuously refine the projection tool with regards to the number of supported projection types, the graphical quality of the rendering process and the didactic quality of the accompanying tutorial.

\section{REFERENCES}

Aisch, G. (2014). Kartograph. https://kartograph.org/. Accessed 2018-08-27.

Bostock, M., Ogievetsky, V., and Heer, J. (2011). D3 data-driven documents. IEEE Transactions on Visualization and Computer Graphics, 17(12):2301-2309.

GITTA (2018). Gitta - geographic information technology training alliance. http://www.gitta.info. Accessed 2018-08-27.

Iliffe, J. and Lott, R. (2008). Datums and Map Projections: For Remote Sensing, GIS and Surveying. Whittles Pub.

Snyder, J. (1987). Map Projections-a Working Manual. U.S. Geological Survey professional paper. U.S. Government Printing Office. 